\title{
Ueber den Nervus depressor als Reflexnerv der Aorta.
}

\author{
Von \\ Dr. med. Gr. Köster, \\ Privatdocent an der Universität und \\ Leipzig. \\ Dr. med. A. Tsehermak, \\ Privatdocent an der Universität \\ Halle a. S.
}

Unsere Vermuthung, dass der Nervus depressor, speciell beim Kaninchen, als ein Ast der sensiblen Portion des Nervus vagus seine Zellen im Ganglion jugulare habe, dass er im Aortenbogen endige oder entspringe und einen sensiblen oder Reflexnerv der Aorta, nicht einen solchen des Herzens (C. Ludwig und Cyon) darstelle, hatte schon durch unseren anatomischen Nachweis seiner charakteristisehen Zellengruppe im Ganglion jugulare und seiner feinen Aufzweigung und anscheinenden Endigung in der Aortenwand eine wichtige Grundlage gefunden. Ueber diese Untersuchung haben wir an einem anderen Orte berichtet ${ }^{1}$ ).

Andererseits führte uns jene heuristische Hypothese zu dem Versuche, den N. depressor durch Steigerung des Blutdruckes bei intactem Gefässsystem, dann aber auch durch den künstlichen Injectionsdruck im künstlich isolirten, überlebenden Aortenbogen in Erregung zu versetzen und die elektrische Componente des Erregungsprocesses festzustellen. Es geschah dies durch Beobachtung der negativen Schwankung des Nervenstromes am durchschnittenen Stamme des N. depressor bei Längsquerschnitt-Ableitung. Gleich hier sei vorweggenommen, dass die Mehrzahl unserer Versuche, die mit mancherlei Schwierigkeiten zu kämpfen hatten, positiv ausgefallen ist und uns den Schluss zu gestatten scheint: der N. depressor lässt sich unter günstigen Bedingungen durch Steigerung des

1) Ueber Ursprung und Endigung des Nervus depressor und des Nervus laryngeus superior beim Kaninchen. Arch. f. Anat. u. Pbys., Anat. Abth. von W. His 1902 Suppl. 
Füllungsdruckes oder der Wandspannung der Aorta erregen und erscheint auch auf Grund dieses physiologischen Nachweises, in Analogie zum anatomischen Befunde, als sensibler oder Reflexnerv des Aortenbogens.

\section{Methodik und Ergebnisse der Versuche.}

In der ersten Versuchsreihe (sechs Thiere, elf Einzelversuche) wurde der $\mathrm{N}$. depressor am Halse des Kaninchens ohne Narkose aufgesucht, durch Verfolgen des Stammes bis zu seinem Ursprunge aus dem N. laryngeus superior oder Vagusstamme identificirt und möglichst hoch oben ligirt und durchschnitten. Wir trennten den N. depressor gewöhnlich auf eine längere Strecke vom Sympathicus; in der II. Versuchsreihe wurde mitunter zur Beschleunigung auf diese Isolirung verzichtet und beide Nervenstämme vereint auf eine längere Strecke herausgehoben. Nach Anbringen eines reinen Querschnittes ward der Nerv auf unpolarisirbare Thonspitzenelektroden (Strecke 2--4 mm) aufgelegt und der Längsquerschnittstrom zum Galvanometer abgeleitet. Das Nachbargewebe war durch einen Glasstab von den Elektroden ferngehalten. Wir bedienten uns durchwegs des hochempfindlichen Deprez-d'Arsonval'schen Solenoidgalvanometers Typ. III der Firma Siemens \& Halske, dessen Empfindlichkeit (innerer Widerstand mit Vorschaltwiderstand $10000 \mathrm{Ohm}$ ) auf $1 \mathrm{~mm}$ Ablenkung bei $1 \mathrm{~m}$ Beobachtungsentfernung für $\frac{8,5}{10^{-10}}$ Ampère indieirt war, in speciellen Messungen seitens Herrn Professor J. Bernstein aber noch etwas grösser befunden wurde, nämlich $1 \mathrm{~mm}$ Ablenkung bei $1 \mathrm{~m}$ Beobachtungsentfernung für $\frac{7,8}{10^{-10}}$ Ampère. Der Beobachtungsabstand von Fernrohr und Galvanometer war zu $3 \mathrm{~m}$ gewählt.

Wir liessen im Allgemeinen den schwachen Nervenstrom uncompensirt. Während der eine von uns am Fernrohre beobachtete und die Ablesungen notirte, führte der andere auf des ersteren Geheiss die Compression der vorher schon durch die Bauchdecken durchpalpirten Aorta abdominalis aus. Die Compression wurde durch 2-5 (ausnahmsweise bis 8) Secunden ausgeführt. Bei längerer Ausübung des Druckes traten störende Krampfbewegungen des Thieres auf, welche durch Abgleiten des Nerven von den Elektroden u. A. 
manchem Versuche ein vorzeitiges Ende setzten. Bei gutem $\mathrm{Zu}$ : stande des Nerven (frisch präparirt, eventuell neuer Querschnitt) und bei Ausbleiben sonstiger zufälliger Störungen war regelmässig eine: deutliche Abnahme des Längsquerschnittstromes im Anschlusse an Compression und Blutdrucksteigerung und ein Wiederansteigen des: Stromes, öfters scheinbar zu grösserer Höhe wie zuvor, nach Aufheben der Compression, also während des Druckabfalles, zu beobachten. Das Ausmaass der negativen Schwankung betrug $0,5-1-6,5$ Scalentheile. Unter dem prompten Rückgange nach Aufhebung der Compression häufig über den Ausgangspunkt hinaus scheint sich eine zweite, positive Schwankung zu verbergen. Doch erscheint uns der Nervus depressor als kein geeignetes Object zum Entscheid dieser Frage.

Der Versuch wurde in jedem Falle öfters wiederholt und lieferteeventuell zehn Mal hintereinander dasselbe Resultat, bis das fortschreitende Absterben des Nervenstammes oder absichtliches Aufgeben der Beobachtung ein Ende setzten. . Von den elf Versuchen fielen sieben positiv, vier negativ aus, und zwar zwei an dem erst an zweiter Stelle vorgenommenen Nerven. Vor dem spontanen Erlöschen jedes Effectes wurde nicht selten eine Beschleunigung des spontanen Absinkens des Nervenstromes während der Compression ohne folgenden Wiederanstieg beobachtet (vgl. Aehnliches unter Versuchsreihe II). - Nachdem beide Depressoren der Beobachtung unterzogen waren, wurde die Wunde gesäubert und geschlossen und das Thier überleben gelassen. Das Detail ist aus den Versuchsprotokollen im Anhange (Reihe I) zu ersehen. Das Ergebniss dieses einfachen Versuches ist ein so typisches, dass wir nicht anstehen, denselben, der bei Curaresirung und künstlicher Athmung des Thieres noch leichter ausführbar ist, als Vorlesungsexperiment zu empfehlen.

In der zweiten Versuchsreibe (elf Thiere, 22 Einzelversuche) wurde die Präparation und Ableitung des N. depressor in derselben Weise vorgenommen, wie oben beschrieben. An die Nervenpräparation schlossen wir aber unter möglichster Beschleunigung folgende Operation ohne Narkose an. Nach Spaltung der Haut in der Medianlinie wurde das Sternum mit Hohlsonde und Arterienhaken umstochen und die Arteriae mammariae int. en masse doppelt ligirt, das Sternum durchschnitten und dessen untere Hälfte mit den Rippenansätzen in genügender Ausdehnung resecirt. Nach Spaltung des Herzbeutels ward ein starker Faden um die Wurzel der Gefässe, incl. Aorten- 
wurzel, herumgeschlungen und festgeknotet und das pulsiriende Herz abgetrennt. Nach Wegtupfen des ausgeströmten Blutes wurde in die Aorta descendens centralwärts eine Canüle eingebunden. Das in situ belassene Präparat bestand demnach aus dem central blindgeschlossenen Aortenbogen und den bis zu diesem absteigenden N. depressores.

Während des Auflegens des einen Depressorstammes und der Einstellung des Galvanometers setzte der eine von uns eine mit $0,6 \%$ iger Kochsalzlösung von $39^{\circ}$ oder Zimmertemperatur gefüllte Spritze von $100 \mathrm{ccm}$ an die Canüle an und trieb dann auf Geheiss des Fernrohrbeobachters bei "ruhigem". Stande des Galvanometers in rhythmischen Stössen die Pulswelle immitirend die Flüssigkeit in den Aortenbogen ein. Die Injection wurde durch 3-10" vorgenommen und mit Rücksicht auf die öftere Wiederbolung des Versuches sistirt, sobald ein deutlicher Effect wahrzunehmen wảr. Die injicirte Lösung drang durch die Subclaviae in die Extremitäten, durch die Carotiden (mitunter ligirt) und Vertehrales in das Gehirn und Rückenmark: da durch die Massenligirung der Wurzel aller Gefässe am Herzen der Ausweg aus der Cavae gesperrt war, sammelte sich die Injectionsmasse in den Venen, speciell in den Gefässen des Ahdomens an, welches am Schlusse wiederholter Einspritzungen stark aufgetrieben war.

Trotz thunlichster Beschleunigung der ganzen, nicht einfachen Operation, die immerhin bis zum ersten Injectionsversuch ca. $30^{\prime}$ dauerte, war in der Hälfte der Fälle - wohl in Folge raschen Absterbens der Depressorneuronen, etwa speciell ihrer Endigungen sowie in Folge eventueller Läsion des Depressorstammes - das Versuchsergebniss negativ. Besonders war dies an dem an zweiter Stelle benutzten Depressor der Fall (vgl. die Thiere IV, VIII, XI). Den elf negativen Fällen (Versuch IV B, V A und B, VI A und B, VII A und $\mathrm{B}$, VIII $\mathrm{B}$, IX A und $\mathrm{B}, \mathrm{XI} \mathrm{B}$ ) stehen ebenso viele positive gegenüber. Als Zeit für das Ueberleben des Depressors unter den gewählten Bedingungen können wir auf Grund unserer Erfahrungen etwa $10-70^{\prime}$ nach dem Herztode des Thieres angeben, ohne dass in den einzelnen Fällen ein Grund für diese starken Verschiedenheiten aufzufinden wäre. Von dem N. vagus, der sich bis dahin in situ befunden hatte und eben erst herausgenommen war, erhielten wir mehrfach noch einen starken Nervenstrom (eventuell sogar über 
die Scala hinaus), wenn die isolirten Depressoren keinen solchen mehr aufwiesen.

Das Ausmaass der negativen Schwankung variirte in den einzelnen Versuchen, auch bei den verschiedenen Injectionen ziemlich erheblich. Während z. B. in Versuch X A Injection 7 die negative Phase 2 Scalentheile ausmachte 542

$\begin{array}{ll}540 & \text { Injection } \\ 550 & \text { Schluss }\end{array}$

betrug sie in Versuch III A Injection 717 Scalentheile

599

582

Injection

618

Schluss

und im gleichen Versuch bei der 5. Injection gar 94 Scalentheile, nämlich 601 . Injection

521

599

Beim Zurückgehen wurde der Ausgangspunkt nicht selten überschritten: unter dieser Erscheinung scheint sich eine zweite, positive Schwankung zu verbergen (z. B. Versuch III A Injection 7 und X A Injection 7) ${ }^{1}$ ).

Gerade der Umstand, dass der galvanische Effect im Laufe des Versuches mit dem spontanen Absinken des Nervenstromes abnahm und schliesslich verschwand, dass er in der Hälfte der Versuche ganz ausblieb, scheint uns mit dafür zu sprechen, dass in den positiven Fällen (11) thatsächlich eine negative Schwankung des Depressorstromes zur Beobachtung kam, also die Depressorendigungen im Aortenrohre durch den Füllungsdruck erregt wurden. - Im gleichen Sinne lässt sich folgende wiederholt gemachte Beobachtung verwerthen (z. B. Versuch III A, Injection 11 und 12). Bei schon niedrigem und wejter sinkendem Nervenstrome veranlasste die Injection nur ein rascheres Absinken

1) In einem nicht registrirten Versuche, in welchem wir von dem am Depressorstamme belassenen Ganglion jug. vagi ableiteten, schien überhaupt nui eine positive Schwankung vorzukommen. 
des Stromes; nach Anlegen eines neuen Quersehnittes hingegen war der Nervenstrom stärker und liess eine deutliche negative Schwankung mit folgendem Wiederanstieg beobachten, nämlich :

$\begin{array}{lllll}597 & & 608 & \\ 587 & \text { Injection 11 } & & \text { Injection } 12 \\ & \text { Schluss } & & 592 & \text { Sehluss } \\ 585,0 & & 599 & \\ 585,5 & & 593 & \\ & & 589 & \end{array}$

Dass der Modus des mechanischen Reizes entscheidend ist, geht aus mehreren Beobachtungen hervor, denen zu Folge die künstliehe Längendehnung des leeren Aortenrohres durch $\mathrm{Zug}$ in Gegensatze zur rhythmischen Querausdehnung durch Füllungsdruck ohne wahrnehmbaren Effect blieb. Die Ungleichwerthigkeit jener beiden Formen mechanischer Einwirkung wird u. A. illustrirt durch die Versuche mit Zug durch Hebelbelastung und mit Füllungsdruck am isolirten Kaltblüterherzen [Ransom ${ }^{1}$ ) am Octopus mit Füllungsdruck, Schoenlein ${ }^{2}$ ) an Aplysia mit Zug durch Hebelbelastung, Straub ${ }^{3}$ ) an Aplysia mit Füllungsdruck]. Man vergleiche ferner die von J. P. Pawlow begründete Idee einer weitgehend electiven Reizbarkeit der verschiedenen Nerven bezw. Nervenendigungen des Darmtractus.

Dass es gelang, den Depressor innerhalb der Aortenwand durch den elektrischen Reiz (Inductionsströme) zu erregen und dabei eine negative Schwankung des Nervenstromes zu beobachten, sei nur nebenbei erwähnt.

Das Detail unserer Versuche ist aus den Protokollen im Anhange (Reihe II) zu ersehen.

\section{Schlussbetrachtung.}

Nach dem Vorstehenden weisen uns das physiologische Experiment sowie die histologische Untersuchung auf die Aorta als das Endziel des N. depressor hin. Eine weitere Beziehung des N. depressor zum Herzen selbst wird zwar durch den physiologiseben Versuch nicht ausgeschlossen, wohl aber spricht der anatomische

1) Journ. of phys. t. 5 p. 261 spec. 271. 1883.

2) Zeitschr. f. Biol. Bd. 12 S. 187. 1894.

3) Pflüger's Arch. Bd. 86 S. 504. 1901. Vgl. auch A. Tschermak, Ueber den Einfluss localer Belastung auf die Leistungsfähigkeit des Skeletmuskels. Pflüger's Arch. Bd. 91 s. 217. 1902. 
Befund gegen eine solche Möglichkeit. Unsere erste Versuchsreihe that nur die Erregbarkeit des N. depressor durch Blutdrucksteigerung überbaupt dar, die zweite hingegen erweist die Aorta als einen Angriffsort des Wandspannungsreizes.

Die Ludwig-Cyon'sche Theorie von der Erregung des N. depressor durch gesteigerten Blutdruck und von seiner Ventilfunction als depressorischer Reflexwerv hat in letzter Zeit durch eine Beobachtung von J. P. Pawlow ${ }^{1}$ ) eine gewichtige Stütze gefunden. Derselbe constatirte nämlich, dass bei hohem Blutdrucke die Durchtrennung beider Depressoren am Kaninchen einen weiteren Anstieg des Blutdruckes zur Folge hat, dass also in jenem Falle der N. depressor wirklich einen depressorisehen Tonus besitzt, sozusagen eine Ventilfunction ausübt. - Unsere Feststellung, dass Blutdrucksteigerung am intacten Gefässsystem, ja auch künstlicher Füllungsdruck am isolirten Aortenbogen den N. depressor zu elregen vermag, fügt sich hier an als ein weiteres Argument für die Ludwig-Cyon'sche Depressorlehre. Wir glauben nur den Aortenbogen, nicht das Herz als Angriffsort des Druckreizes betrachten zu sollen. - Reizbarkeit gewisser Vagusfasern für die Steigerung der Wandspannung demonstrirt der bekannte HeringBreuer'sche Aufblasungsversuch an den Lungen - auf Grund des eintretenden Exspirationsreflexes an den Nasenflügeln und am Zwerchfell. Ein Gleiches lehrt die bekannte Beobachtung E. Hering's, dass mässige Aufblasung der Lungen bei intacten Vagis eine Beschleunigung der Herzthätigkeit zur Folge hat.

Die engste Analogie zu unseren Beobachtungen bieten jedoch die im Hallenser Institute ausgeführten Versuche M. Lewandowsky's ${ }^{2}$ ). Derselbe konnte bei Aufblasung der Lungen eine negative Schwankung des Nervenstromes am peripheren Vagusstamme constatiren.

Wir fühlen uns verpflichtet, auch an dieser Stelle Herrn Prof. J. Bernstein für seine vielfache Förderung unserer Untersuchung und die Ueberlassung der Arbeitsmittel des Hallenser Institutes unseren besten Dank auszusprechen.

1) Mitgetheilt auf dem Congresse russischer Naturforscher und Aerzte in St. Petersburg. December 1901.

2) Zur Lehre vom Lungenvagus. Beobachtungen über Schwankungen des Vagusstromes bei Aenderungen des Lungenvolums. Diss. Halle a. S. 1898. 
Anhang.

Versuchsprotokolle*

Reibe I.

Versuch I. A. Depressor sinister (positiv).

1) 514

512 Compression
515
513,5
514

4) 510,5

Compression 508

510

Schluss

7) 510

$\begin{array}{ll}508 & \text { Compression } \\ 510 & \text { Schluss }\end{array}$

10) , 506,5 508 506 505 Schluss
2) 514

511,5 Compression
514,5
514

5) 509

$\begin{array}{ll}507 & \text { Compression } \\ 510 & \text { Schluss } \\ 509 & \end{array}$

8) 510

$\begin{array}{ll}506 & \text { Compression } \\ & \text { Schluss } \\ 510 & \\ 509 & \end{array}$

11) 501

Compression
3) 513 510,5 Compression
515
514,5
513
511,5

6) 510 507,5 Compression
509
508

9) 504 501,5 Compression
506,5
504

12) 498 $\begin{array}{ll}497 & \text { Compression } \\ 499 & \text { Schluss } \\ 497 & \end{array}$
13) 496,5

$\begin{array}{ll}496 & \text { Compression } \\ 497 & \text { Schluss }\end{array}$

B. Depressor dexter (positiv).

1) 449 449 Compression Schluss 449

4) 449 446 Compression 447 Schluss
2) Neuer Querschnitt 453 Compression Schluss 451

5) Neuer Qucrschnitt 481,5 479 Compression 
7) 460 463 460,5 460
8) 457,5 456,5 Compression
456,5 $10-13$ negativ
9) 455

$\begin{array}{ll}453 & \text { Compression } \\ 453 & \text { Schluss }\end{array}$

Versuch II. A. Depressor dexter (durchwegs negativ).

B. Depressor sinister (durchwegs negativ).

Versuch III. A. Depressor sinister (positiv).

1) 435

\begin{tabular}{l}
437 Compression \\
\multicolumn{1}{c}{ Schluss } \\
435,5 \\
437 \\
439
\end{tabular}

4) 449

$\begin{array}{cl}455,5 & \text { Compression } \\ 446 & \text { Schluss } \\ \vdots & \text { Abfallen d.Ner- } \\ & \text { ven in Folge } \\ & \text { Unruhe des } \\ & \text { Thieres }\end{array}$

7) 497

$\begin{array}{ll}501 & \text { Compression } \\ 496 & \text { Schluss }\end{array}$

10) 500 ${ }_{500}^{501,5}$ Schluss .

13) Neuer Querschnitt 512 512 Compression Schluss 512

16) 457

461 Compression
454,5 Schluss
457

2) 438,5

442,5 Compression
439,5 Schluss
443

3) 444

$$
\begin{aligned}
& 449,5 \text { Compression } \\
& 444 \\
& 449
\end{aligned}
$$

5) 498

$\begin{array}{cl}503 & \text { Compression } \\ 496 & \text { Schluss } \\ & \end{array}$

8) 499

$$
\begin{aligned}
& \text { 502,5 Compression } \\
& 495,5 \text { Schluss } \\
& 500
\end{aligned}
$$

11) 502

$$
\begin{aligned}
& 502,5 \text { Compression } \\
& 501
\end{aligned} \text { Schluss }
$$

6) 499

$\begin{array}{ll}502 & \text { Compression } \\ & \text { Schluss } \\ 495 & \\ 499 & \end{array}$

9) 499,5 Compression 501,5 Schluss 500

12) Never Querschnitt 499 $\begin{array}{ll}499 & \text { Compression } \\ 499 & \text { Schluss }\end{array}$

14) Neuer Querschnitt, 15) 438 $\begin{array}{cl}438 & \text { Compression } \\ 438 & \text { Schluss } \\ \vdots & \text { Abfallen d. Ner- } \\ & \text { ven in Folge } \\ & \text { Unruhe des } \\ & \text { Thieres }\end{array}$

18) 461,5

17) 457

$$
{ }_{463,5-}^{\text {Compression }}
$$$$
\text { Compression }
$$$$
466 \text { Schluss }
$$$$
461,5
$$ 
B. Depressor dexter (in Folge Difformität - nur auf eine ganz kurze Strecke isolirt vom Vagus verlaufend - unbrauchbar).

Versuch IV. A. Depressor dexter (positiv - sehr dünn).

1) 516

$\begin{array}{ll}516 & \text { Compression } \\ 516 & \text { Schluss }\end{array}$

2) 514,5 514,5 Compression
514,5

3) Neuer Querschnitt 495,5 498 Compression Schluss

5) 511

Compression

Schluss

B. Depressor sinister (positiv).

1) 452

$\begin{array}{ll}452 & \text { Compression } \\ 452 & \text { Schluss }\end{array}$

4) 447,5

$$
\begin{aligned}
& 449,5 \text { Compression } \\
& 447,5 \text { Schluss } \\
& 4 \text { Abfallen des } \\
& \text { Nerven }
\end{aligned}
$$

7) 414 416,5 Compression Schluss 416 417
2) 452 $\begin{array}{cc}453 & \text { Compression } \\ \vdots & \text { Abfallen des } \\ & \text { Nerven }\end{array}$

5) Neuer Querschnitt 402,5 405,5 Compression
407

8) 419,5 421 Compression
419,5

10) 440 $\begin{array}{cc}440 & \text { Compression } \\ \vdots & \text { Abfallen des } \\ & \text { Nerven }\end{array}$
3) Neuer Querschnitt 448 449,5 Compression 448 Schluss

6) 413 414,5 Compression
413,5

9) 420 420,5 Compression
$\vdots \quad$ Abfallen des
Nerven

Versuch V. A. Depressor sinister (positiv).

1) 329,5 Compression 325,5 Schluss
2) 325,5

322,5 Compression
324,5
320

E. Pflüger, Archiv für Physiologio. Bd. 93.

3) 318,5

315 Compression
321,5
317 
4) 315,5

313 Compression
$\quad$ Schluss
318
311,5
314

7) 363,5 361,5 367,5
Compression Schluss

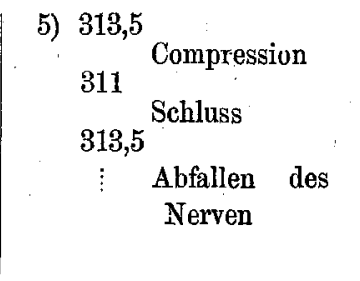
8) 357,5

$\begin{array}{ccc}355 & \text { Compression } \\ & \text { Schluss } & \\ 357 & & \\ \vdots & \text { Abfallen } & \text { des } \\ & \text { Nerven } & \end{array}$

6) Neuer Querschnitt 370,5 Compression 365 durch $5 "$ $370,5^{\text {Schluss }}$ 368

9) 361 Compression 357 durch 4" 359,5 Schluss 358

10) 355

Compression
durch 8"
351,5 Schluss
355 Schluss des
$\vdots \quad \begin{gathered}\text { Abfallen } \\ \text { Nerven }\end{gathered}$

B. Depressor dexter (negativ - ansserordentlich dünn).

2) Neuer Querschnitt 308,5 308,5 Compression
309

Versuch VI. A. Depressor sin ister (durchwegs negativ - dünn).

B. Depressor dexter (positiv).
1) 310,5 309,5 Compression
310,5
Abfallen des Nerven

2) Neuer Querschnitt 309 Compression 306,5 308,5
307 4-9 negativ.
3) 307,5 Compression
305,5 Schluss
307,5 Shallen des
$\vdots \quad \begin{gathered}\text { Abfallen } \\ \text { Nerven }\end{gathered}$

\section{Reihe II.}

Versuch I. A. Depressor dexter (positiv).

B. 'Depressor sinister (positiv).

An jedem Nerven war innerhalb 40 Min. bei jeder Injection eine negative Schwankung des Nervenstromes bis zu vier Scalentheilen zu beobachten. Der- 
selben folgte ein Wiederansteigen ohne Erreichen des Ausgangspunktes. Nach 40 Min. gaben beide Nerven keinen Effect mehr.

Versuch II. A. Depressor dexter (positiv).

B. Depressor sinister (positiv).

Herausgegriffene Beispiele:

1) 790

$\begin{array}{ll}738 & \text { Injection } \\ 745 & \text { Schluss }\end{array}$

2) 840

$\begin{array}{cc}800 & \text { Injection } \\ 810 & \text { Schluss } \\ & \end{array}$

3) 794

775 Injection

Zerren an der Aorta ohne Effect.

Elektrische Reizung des Aortenbogens gibt eine negative Schwankung von 5-7 Scalentheilen.

Versuch III. A. Depressor sinister (positiv).

\begin{tabular}{|c|c|}
\hline 720 & \\
\hline 715 & Injection \\
\hline 714 & \\
\hline 710 & Schluss \\
\hline 710 & \\
\hline
\end{tabular}

4) Fehlerhaft

7) 599

$\begin{array}{ll}582 & \text { Injection } \\ & \text { Schluss } \\ 618 & \\ 603 & \\ & \end{array}$

10) 610

$\begin{array}{ll}606 & \text { Injection } \\ 600 & \\ 598 & \\ & \text { Schluss } \\ 599 & \\ 593 & \end{array}$

13) 585

$\begin{array}{ll}582 & \text { Injection } \\ 598 & \text { Schluss } \\ 590 & \end{array}$

2) 698

$\begin{array}{ll}696 & \text { Injection } \\ 699 & \text { Schluss } \\ & \\ & \end{array}$

5) 601

$\begin{aligned} & \text { Injection } \\ 507 & \\ 521 & \\ 599 & \text { Schluss }\end{aligned}$

8) 606

$\begin{array}{ll}605 & \text { Injection } \\ 620 & \text { Schluss } \\ 601 & \end{array}$

11) 597

$$
\begin{array}{ll}
587 & \text { Injection } \\
585 & \text { Schluss } \\
585,5 & \\
583 &
\end{array}
$$

14) 585

$\begin{aligned} 583 & \text { Injection } \\ 583 & \text { Schluss } \\ 610 & \\ 605 & \end{aligned}$

3) Neuer Querschnitt 613 Injection 610 619 Schluss 614 609

6) Fehlerhaft

9) 613 $\begin{array}{ll}609 & \text { Injection } \\ 620 & \text { Schluss } \\ 615 & \\ 610 & \end{array}$

12) Neuer Querschnitt 608 592 Schluss 599 593 589

15) 610

$$
\begin{aligned}
610 & \text { Injection } \\
610 & \text { Schluss }
\end{aligned}
$$


B. Depressor dexter (positiv).

1) 622 621

629

624
2) 632

$\begin{array}{ll}630 & \text { Injection } \\ & \text { Schluss } \\ 634 & \\ 632 & \end{array}$

3) 632

$\begin{array}{ll}631 & \text { Injection } \\ 630 & \text { Schluss }\end{array}$

Versuch IV. A. Depressor dexter (positiv).

1) 732

728

722

722

Injection

Schluss

4) 685

683

687

688
2) 699

$\begin{array}{ll}696 & \text { Injection } \\ 697 & \text { Schluss }\end{array}$

5) 687

685,5
686,5 Schluss

3) 693

689 Injection
690,5

6) 680

679

Injection

Schluss

B. Depressor sinister (durchwegs negativ).

Versuch V. A. Depressor dexter (durchwegs negativ).

B. Depressor sinister (durchwegs negativ).

Beobachtung erst relativ spät nach dem Herztode begonnen.

Versuch VI. A. Depressor dexter (durchwegs negativ).

B. Depressor sinister (durchwegs negativ).

Beobachtung erst relativ spät nach dem Herztode begonnen.

Versuch VII. A. Depress or dexter (durchwegs negativ).

B. Depress or sinister (durchwegs negativ).

Versuch VII. A. Depressor dexter (positiv).

1) 320

322,5 Injection

320
2) 324

326,5
323
325 Schjection

3) 333 333,5
330
333

4, 5 negativ

B. Depressor sinister (durchwegs negativ).

Versuch IX. A. Depressor dexter (durchwegs negativ).

B. Depressor sinister (durchwegs negativ). 
Versuch X. A. Depressor sinister (positiv).
1) 724,5
725 Injection
Schluss
745
722
2) 722
720 Injection
20 Schluss 717 712,5 Schluss
3) 717
4) 711
711
Schluss
709 Injection
5) 709
$\begin{array}{ll}707 & \text { Injection } \\ 708 & \text { Schluss } \\ 705 & \end{array}$
6) 702
700 Injection 703,5 Schluss
7) Neuer Querschnitt 542
540 Injection
Schluss
8) 549
$\begin{array}{ll}530 & \text { Injection } \\ & \text { Schluss } \\ 532 & \end{array}$
9) 540,5
528 Injection
599 Schluss 550
10) 530,5
Schluss 520
Injection 499 509
Injection Schluss
11) 517
12) 508

\begin{tabular}{l}
497 Injection \\
\multicolumn{2}{c}{ Schluss } \\
516,5 \\
515,5
\end{tabular}
13) 516,5

512 Injection
521,5
514 Schluss
14) Neuer Querschnitt 627 626,5 Injection

B. Depressor dexter incl. Sympathicus (positiv).

1) 765

$$
\begin{aligned}
765 & \text { Injection } \\
750 & \\
762 & \text { Schluss } \\
750 &
\end{aligned}
$$

4) 750 736,5 740

Schluss

Injection 
38 G. Köster und A. Tschermak: Ueber den Nervus depressor etc.

Versnch XI. A. Depressor dexter incl. Sympathicus (positiv).

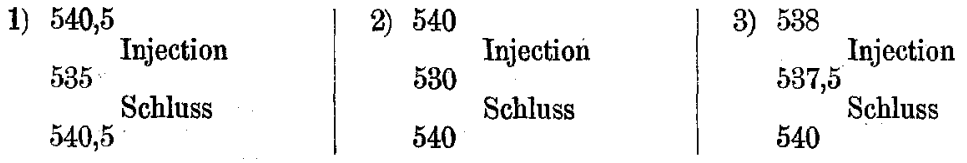

Die folgenden Injectionen fehlerhaft, nach Beseitigung des Fehlers kein Effect mehr zu erzielen.

B. Depressor sinister incl. Sympathicus (durchwegs negativ). 